\title{
Video-based Online Learning: The Other Side of the Looking Glass
}

\section{Dr. Daniel Takashi Kawano, Rose-Hulman Institute of Technology}

Daniel Kawano is an Assistant Professor of Mechanical Engineering at Rose-Hulman Institute of Technology. He received his B.S. degree in Mechanical Engineering from California Polytechnic State University, San Luis Obispo. He obtained his M.S. and Ph.D. degrees in Mechanical Engineering, with a focus in dynamical systems, from the University of California, Berkeley.

Dr. Benson H. Tongue, University of California, Berkeley

GA Tech 1983-1988, UC Berkeley 1988-present. Author of Principles of Vibration and Analysis and Design of Systems in Motion: Dynamics. 


\section{Video-based Online Learning: The Other Side of the Looking Glass}

\section{Introduction}

Education researchers often allude to fundamental changes taking place within the academic environment, enough so that one would be forgiven a bit of jadedness at any new pronouncement. Recently, however, changes to the academic landscape have been occurring that can truly be viewed as earthshaking, no pun intended.

The catalyst for these changes has been digital technology, which has been following its usual trend of increasing capabilities and decreasing cost as time moves forward. The readily available technologies have matured to a point for which one can deliver significant educational content in ways that are completely divorced from the traditional classroom. Not only can it be delivered, it can be delivered affordably, an aspect that has not escaped the notice of both academic administrators and outside observers.

In our view, this digital boulevard is a thoroughfare that, having been opened for traffic, will not be closing down any time soon. Indeed, the use of screen-capturing and other video technologies as supplemental learning tools is permeating throughout higher education ${ }^{1-3}$. If one accepts the premise that digital delivery in one form or another is here to stay, it is incumbent upon those who create educational content to ensure that it leads to a good pedagogical destination.

In an effort to enrich student learning in dynamics via digital media and interactive tools in a web-based platform, the authors are working to transition a traditional dynamics course into just such a media-rich environment. This dynamics learning platform has been evaluated at the prototype and proof-of-concept stages by various instructors, and the authors have received very valuable and positive feedback from these reviews. However, the authors are keenly aware that the end users for the product are individual students, not professors. Student reactions to the approach will surely differ from their instructors for two reasons. One is the greater digital facility that, in general, students will possess. Along with this facility will come particular expectations regarding content richness and speed of content flow that their older and less digitally immersed teachers may well not share. Second, students will encounter the material from an unlearned perspective, whereas their teachers, being expert in the material, will perceive whether the material is correct but not necessarily whether it is more effectively learned in the new presentation. The difference between these two viewpoints is profound. To express it more concretely, an instructor may wish to understand the behavior of a car's dynamic slip angles as it enters a decreasing radius curve whereas a student is focused more on questions like "Which pedal is the gas and which is the brake?" 
To most effectively deliver learning within a one-way video format, one must stop and ask which elements effectively support and which hinder student performance. There are both technical and cognitive issues to this question that merit examination. As an example, the video itself can be high or low resolution and be presented on a large or small screen. The size of the screen affects learning simply because something too small loses readability. But large images require a large amount of data to be rendered accurately, and this file size has implications for the delivery channel (broadband, WiFi, or cellular). Size and speed problems can be alleviated through image quality reductions but doing so may negatively affect the students' ability to learn.

An element of the overall platform for which the authors have been particularly interested in receiving student feedback regards our screen-captured content, including both lecture material and worked out example problems. Specifically, what techniques for video production do students perceive as important with respect to their learning and which yield an overall positive learning experience? In our paper, the authors present the results of a student assessment of sample screen-captured example problems based on a series of posed questions. With the shift from text-based to video-based learning in mind, some of the survey questions were also designed to assess student reaction to certain pedagogical practices in text and lecture translated to video format, as well as preconceptions regarding delivery of video content.

\section{Learning Evaluation Methodology}

Central to our study was the intent to gather student feedback and not presume from our personal vantage point what would be more or less effective. Students were allowed to view several examples of video content that had a single variable altered across the test cases. For instance, they were shown a video of the same material, but for each of the clips the degree of digital compression differed. What they saw on screen ranged from a high quality, HD-like presentation down to one that was more on the level of a highly compressed YouTube video. Unlike regular video imagery, in our case what the student sees is usually a "blackboard" or "whiteboard" with "chalk" or "marker" equations and explanation, all of which remained static after creation. Thus the effect of the compression artifacts was accentuated, there being no generalized motion to fool the brain into thinking the displayed image is of better quality than it actually was. The students were asked to rate the videos, not just from best to worst, which would have been self-evident, but in more learning-centric terms: "good enough to watch without annoyance," "understandable but not great," "not acceptable" and so forth. 
The student survey was conducted at Rose-Hulman Institute of Technology and at the University of California, Berkeley during the winter quarter and spring semester, respectively, of the 20122013 academic year. Students in our dynamics courses were asked to first watch two screencaptured example problems, each broken into several clips. Sample screenshots from one of the screen-captured example problems are shown in Figure 1.

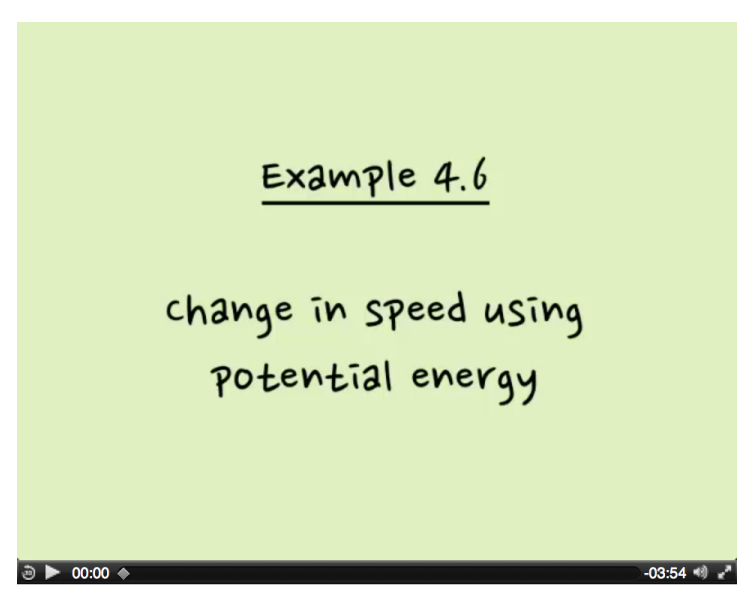

(a)

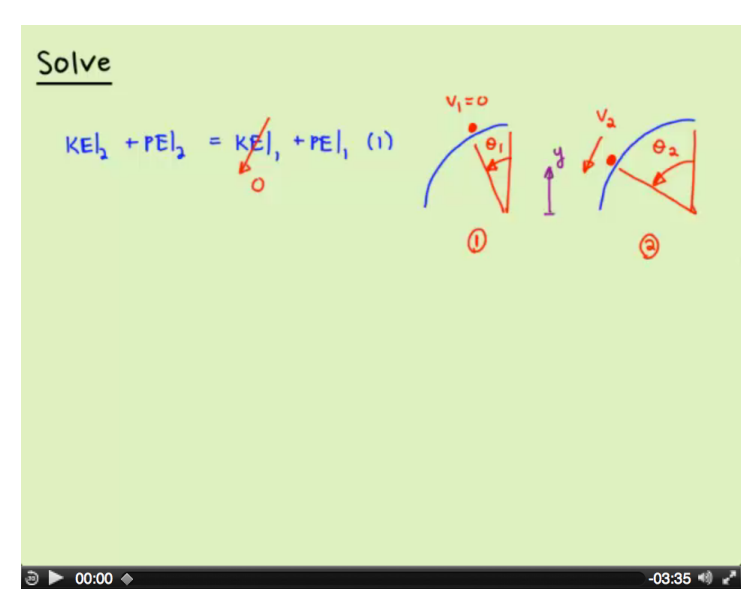

(b)

Figure 1. Snapshots of two clips from the screencast for Example 4.6 used in the study. The first clip (a) contains the introduction screen and the Problem Statement, Goal, Given, Draw, and Formulate Equations sections. The second clip $(b)$ continues the solution process with the Solve and Check sections.

After watching the videos, the students were directed to our institutions' online course management systems to complete a confidential and voluntary survey in which they would rate the importance of various aspects of content delivery with respect to their learning using a sevenpoint Likert scale, as well as provide further explanations and additional comments in text boxes. A total of 58 students participated in the assessment.

\section{Results and Discussion}

The survey results are presented as bar charts in Figures 2 through 7 and in Figures 9 and 10.

\section{Breaking up a solution process}

A practice that is employed to quite varying degrees in engineering texts is the separation of a solution process into components to help guide students through an example in a clear, consistent, and methodical progression, a practice advocated by Gray, Costanzo, and Plesh ${ }^{4}$. In 
fact, they note that the most widely adopted texts on dynamics, even after many years on the market, have yet to adopt a problem solving structure beyond essentially a Problem-Solution format. After employing their method in their dynamics course, Costanzo and Gray concluded that "the use of a structured problem solving approach was beneficial to the students' perception that they were learning the subject matter." In our case, the online dynamics platform the authors are developing is based on the text Dynamics: Analysis and Design of Systems in Motion, 2nd ed., in which example problems are broken into the following steps: Problem Statement, Goal, Given, Assume, Draw, Formulate Equations, Solve, and Check. While a bit different from the approach advocated by Gray, Costanzo, and Plesh, the method we employ has the same spirit. When transitioning from a static text medium to a "live," multi-media-rich environment, is such pedagogical practice, according to students, still of value to their learning?

When asked about the importance of breaking up a solution process (e.g., Goal, Given, Solve, etc.) and identifying the part of the solution process being discussed, the students rated these as being important aspects. Specifically, according to the results in Figure 2, 51 students (88\%) rated breaking up the solution process as important to their learning, with 45 of these students $(88 \%)$ claiming this to be moderately or very important. With respect to identifying the solution process sections, the results in Figure 3 reveal that 52 students $(89 \%)$ rated this as important to their learning, but these students were more evenly split on just how important.

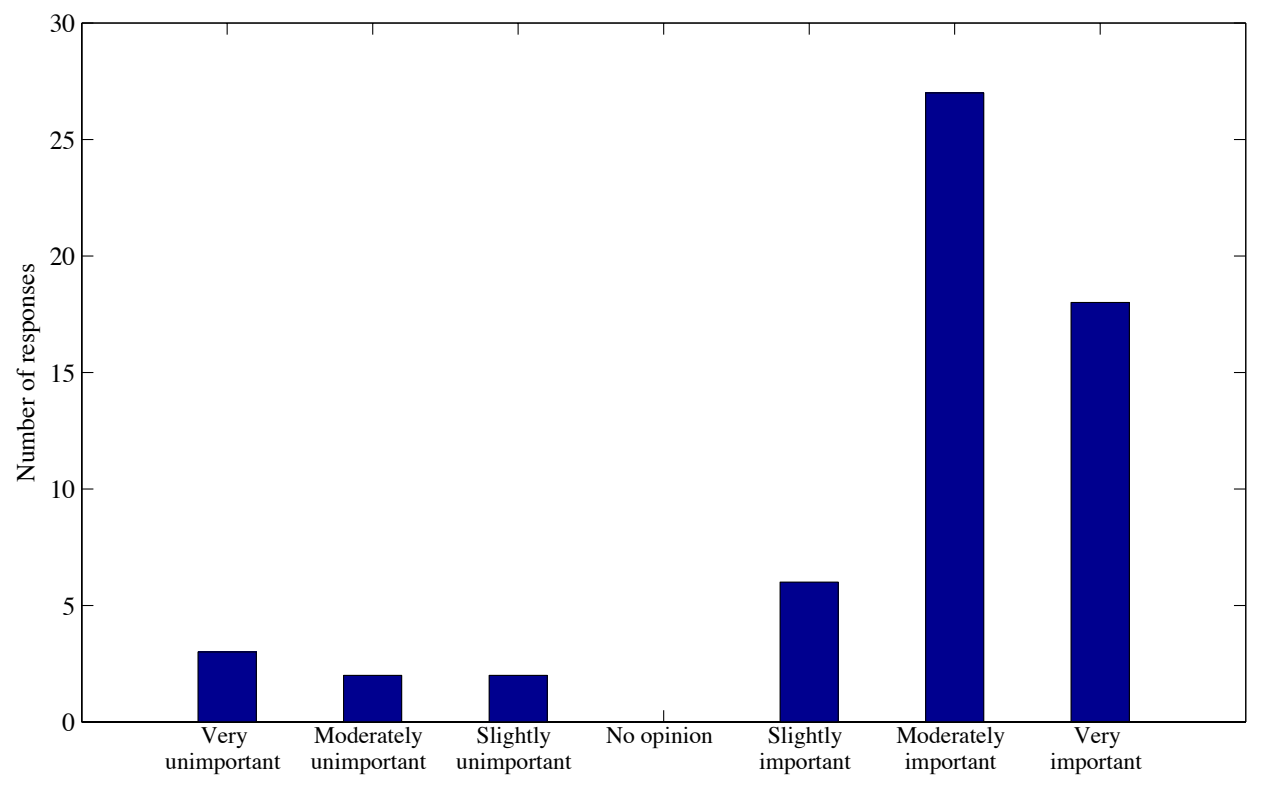

Figure 2. Responses to "Rate the importance of breaking up the solution process (Goal, Given, Draw, etc.) with respect to your learning." 


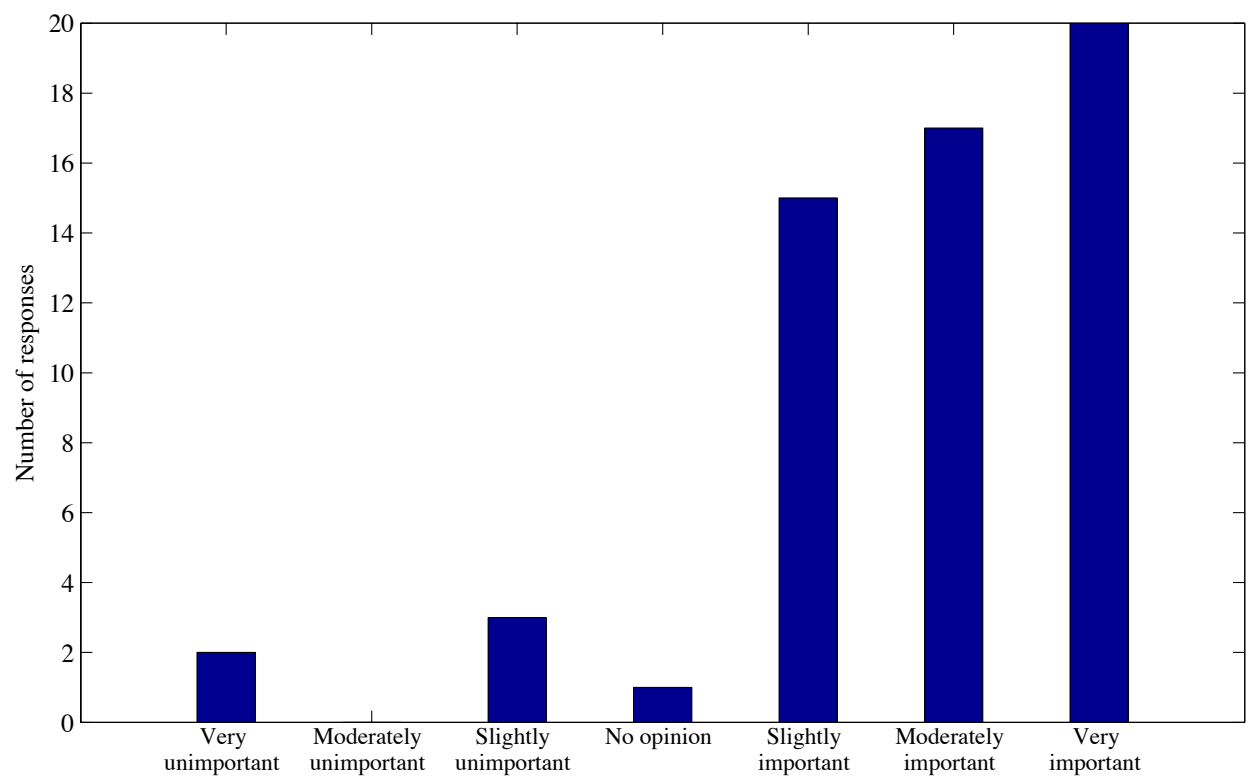

Figure 3. Responses to "How would you rate the importance of including section labels (Draw, Solve, etc.) in identifying the step of the solution process being discussed?"

Overall, the survey results indicate that students overwhelmingly support having the solution process for screen-captured examples broken into sections and having section identifiers visible as the solution process progresses, and thus the authors believe this is a pedagogical practice that ought to be continued when transitioning from print to video.

Coincidentally, a few weeks after completion of this evaluation, one of the authors received an email communication from a prior student who has spent the last few years in the workforce, working across a broad range of responsibilities, including cross team communication and management as well as straight engineering. The student communicated that he found he was better prepared than the bulk of his coworkers for all the tasks he encountered precisely because he had been trained to break problems into recognizable chunks. Having been trained in the Goal, Given, etc. approach, he applied them widely and found it was invaluable in tackling problems well beyond the engineering domain.

\section{Including explanatory narration}

Of course, one element that is missing from a text version of an example problem that is easily implementable in a screen-captured version is voice narration. An advantage of voice narration in screen-captured examples is that precious screen space may be reserved for key equations and solution steps while explanations and commentary may be more efficiently conveyed by voice instead of by writing or projecting them on the screen. While the absence of voice narration 
from a screencast may not impede student learning, do students consider voice narration as added value when it comes to their learning?

When asked about the importance of including explanatory narration in the screencasts and the completeness of this narration, the students rated these aspects as being important to their learning. According to the survey results in Figure 4, the students almost unanimously (56 students, or 97\%) agreed that including some level of narration is important to their learning, with $71 \%(40)$ of these students rating narration as being very important. The results in Figure 5 reveal that having in-depth and complete narration is just as important to the students' learning, with $88 \%$ of the students considering this moderately to very important.

Thus, the students overwhelmingly perceive including explanatory narration as an important factor in their learning of material, and therefore the authors feel it would be wise to include this element in screen-captured videos, even with the extra effort and time required to do so.

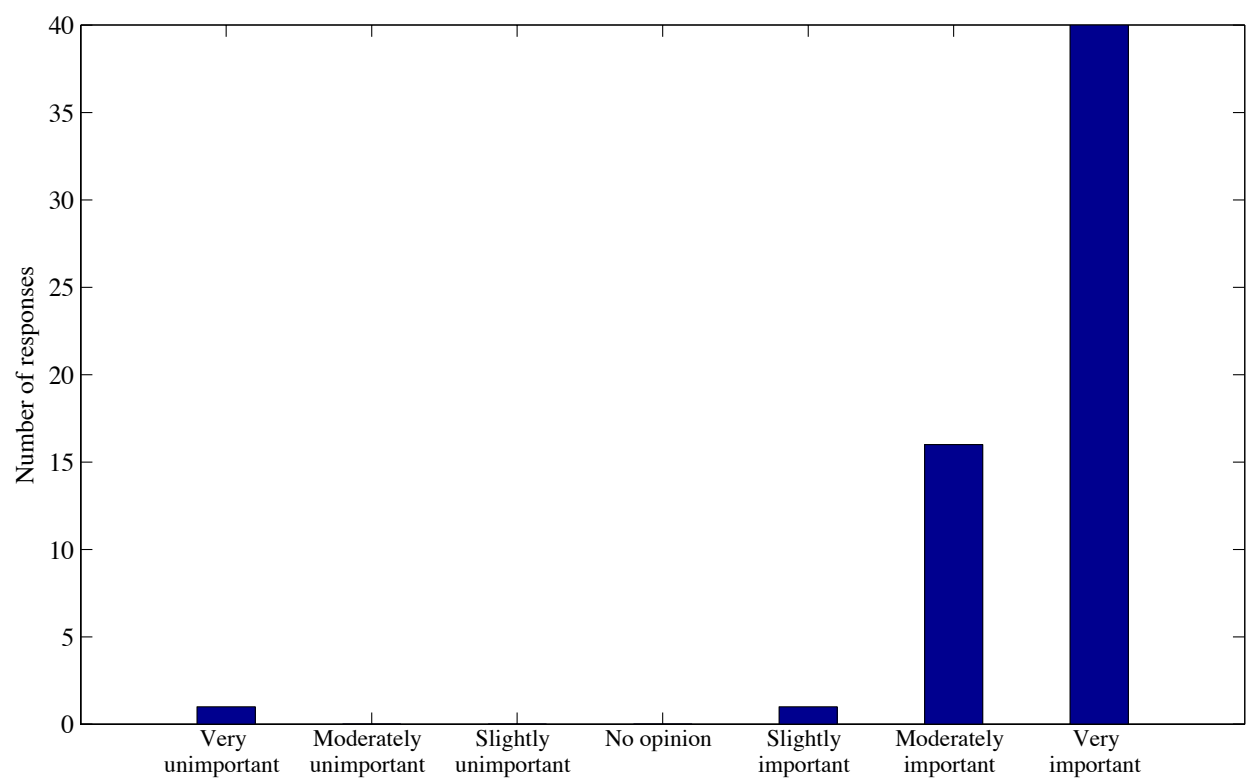

Figure 4. Responses to "How important is it to your learning to have explanatory narration in addition to the written material?" 


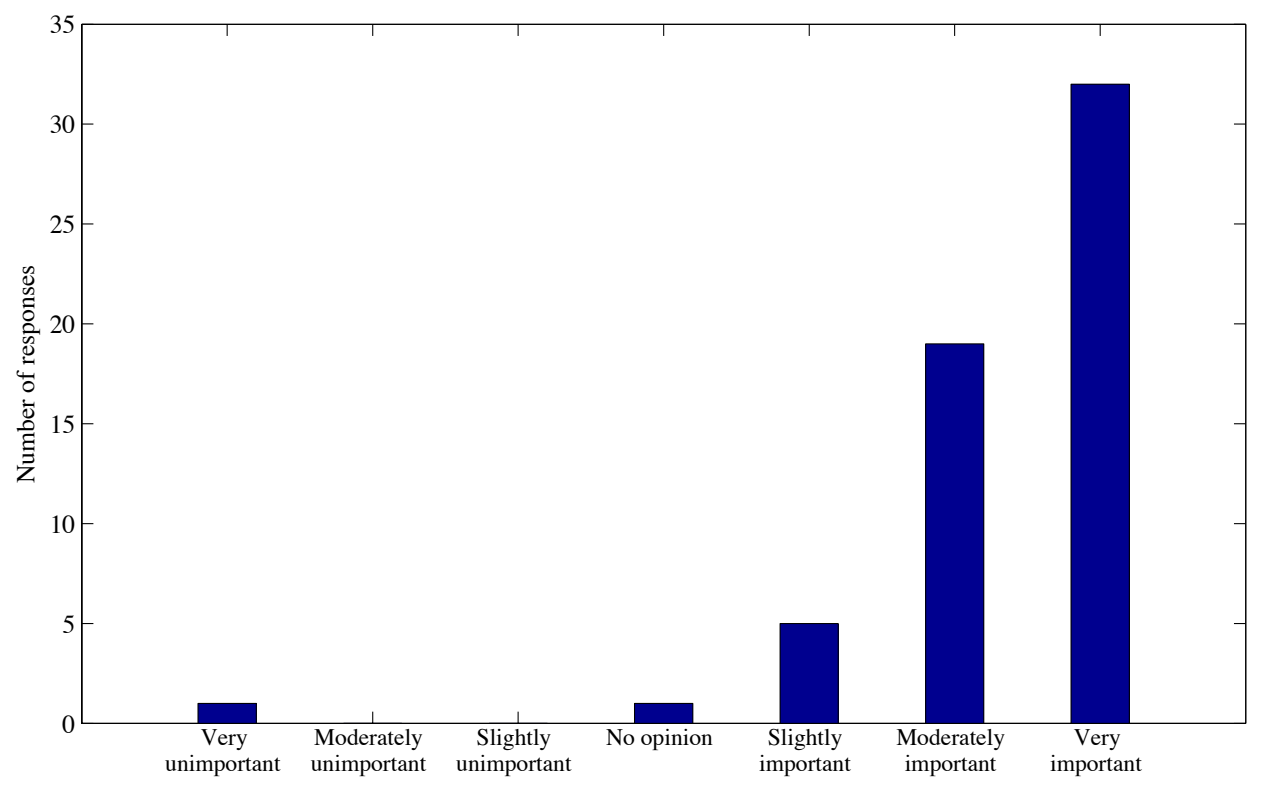

Figure 5. Responses to "To what degree is the depth and completeness of the explanatory narration important to your learning?"

\section{Using multiple colors for text elements}

It is common practice in engineering mechanics texts to use different colors to highlight key words or equations and to differentiate between, say, force vectors (e.g., red) and velocity vectors (e.g., green). However, example problems in textbooks typically do not utilize much color outside of diagrams, that is, equations and other information are generally all one color black. A great advantage of screen-captured examples is that, when coupled with the "right" software for writing on a computer screen with a pen input and manipulating images ${ }^{6,7}$, color may be used effortlessly and more extensively during the solution process in ways that are uncommon in a traditional print medium for various reasons (color-coding text in an example problem solution may be considered unimportant, not worth the effort, not worth the potential increase in publishing costs, etc.). The example videos used during the survey were created with this "multi-color" mindset, using different colors to highlight different text elements (e.g., red for forces on a free-body diagram vs. blue for equations) and to differentiate between key solution steps (e.g., blue for key equations) and simplifications or intermediate steps (e.g., red for crossing terms out and purple for unit conversions). How do students respond to this pervasive but targeted use of color in the videos? 
From a stylistic standpoint, students rated the use of multiple colors for text quite favorably, with 49 students (84\%) rating it as important (see the results in Figure 6). As for whether the students' learning is enhanced by such practice, the survey results in Figure 7 indicate that $66 \%$ (38) of the students responded that their learning is enhanced, while the remaining students were split between being unsure and being certain that their learning is not enhanced.

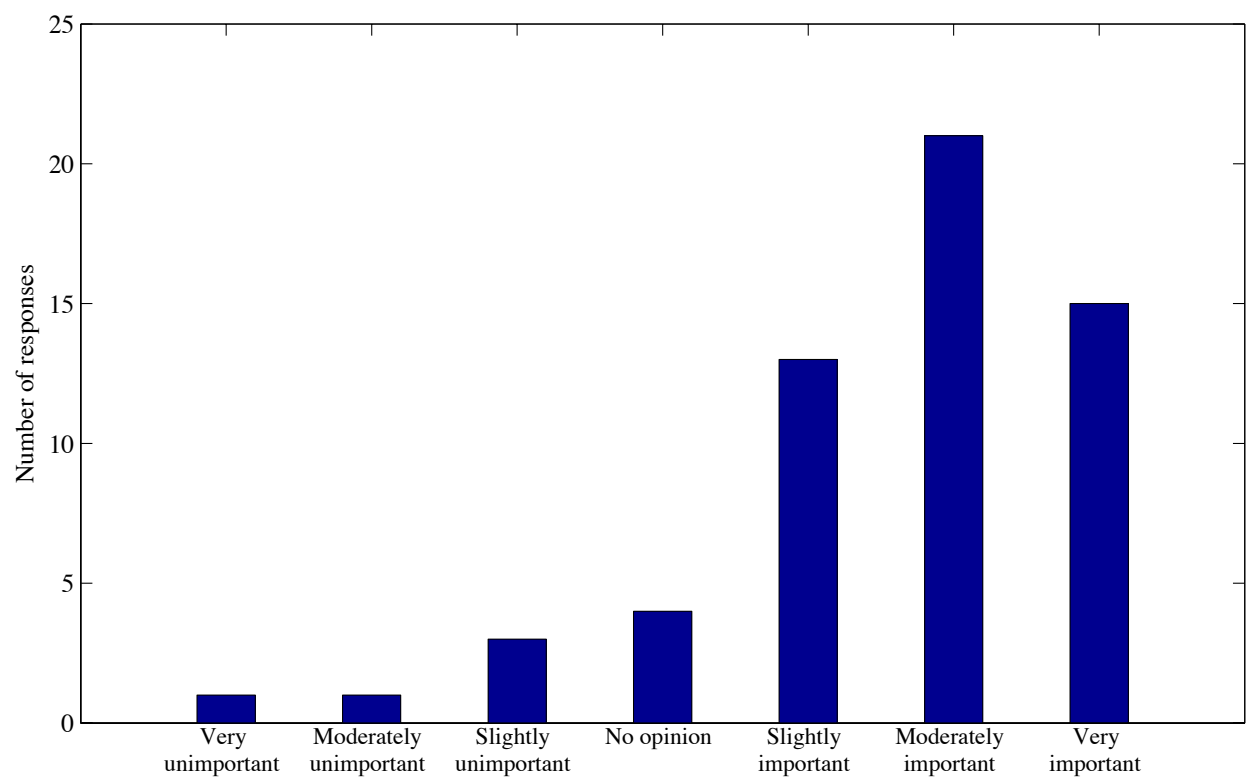

Figure 6. Responses to "Rate the importance of using multiple colors for various text elements."

For those students who responded that their learning is enhanced, the authors sought comments that would help explain their affirmative answer. Specifically, these students were asked to "describe an example that illustrates how your learning is enhanced using different colors for various text elements." A majority of the responses were some variation of the theme that using different colors helped to highlight important parts of the solution process and make distinctions between parts:

- "I liked how the assumption section was a different color than the rest. It showed/meant that it was an important step that should not be confused with or lost in the rest of the problem."

- "It helps me differentiate between different steps and identify common analysis methods." 


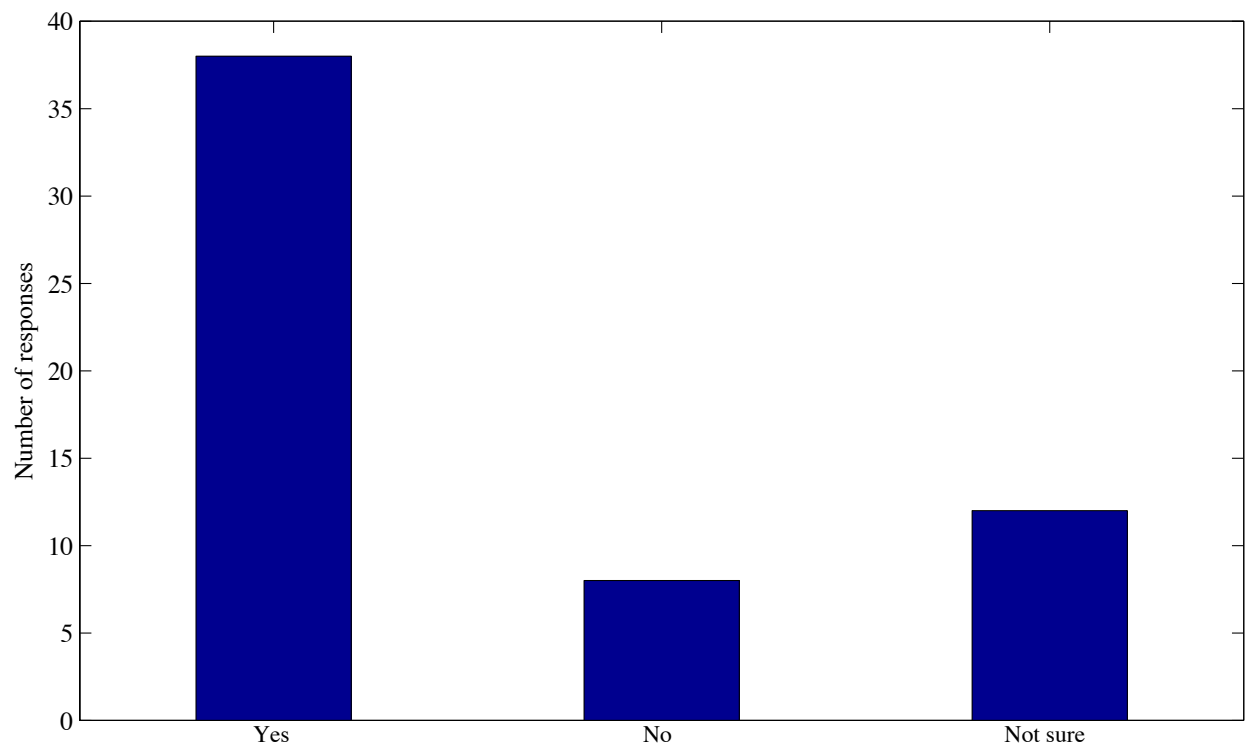

Figure 7. Responses to "Is your learning enhanced by using different colors for various text elements?"

- "Using different colors simply helps me pay attention and I feel more aware of the progression of the problem as it plays out as opposed to simple black and white."

- "It just helps make it clearer what elements are bodylines, dimension lines, projected lines, etc. In formulas, it makes it clearer when a term is being struck or approximated to a certain value."

- "It makes it a lot quicker to understand the solution process if steps were more or less color coded, especially if you don't want to wait for the narration."

With two-thirds of the students responding that targeted use of multiple colors enhances their learning, the authors believe this is a practice that should be implemented in our screen-captured examples.

An interesting point we neglected to ask about is consistency in the use of color. Though some effort was made to use color in some consistent manner in the videos, color usage inevitably drifted over the course of video production. While we did not survey the students about their thoughts on consistent use of color, the students were asked to comment on how color enhanced their learning (if they claimed this was the case), and a few students voluntarily provided feedback on general schemes for color consistency: 
- "box the answer in a different color, put headings in different color, put COLM [conservation of linear momentum] and COAM [conservation of angular momentum] in different colors."

- "Different colors seem to keep me more aware of changes in the information being presented even if the color isn't applied according to any type of systematic regime."

- "If explaining energy, I think it would be useful to have one color text be used for kinetic energy terms and another color be used for potential. Another example could be differentiating between conservative and nonconservative work.

Additionally, if you were going through an example that involved an equation with lots of terms, different colors would help me match the numbers we plug in with the variables in the equation."

- "In the two examples I looked over, I really enjoyed how he used the different colors for the diagrams and the equations. The fact that red was used to cross things out or denote special relations and specific quantities $(\mathrm{g}=32.2 \mathrm{ft} / \mathrm{s}$, etc.) made the material a lot more informative and easier to grasp ... The use of different colors is great, but should be limited to around 2 to 3 or 4 . Any more than that and it may seem to jumbled and could be harder for the student to grasp the concept fully."

- "Given that the narrator goes over the problem statement and addresses each aspect of the problem in turn (e.g. assumptions, given, goal, and etc.), it helped that he color-coded each term based on its category."

What we take away from these comments is that while one student said a systematic approach to color usage may not be necessary, others claim that the video content could be improved by utilizing some consistent approach, e.g., different colors for different parts of the solution process and different types of equations, or different colors for terms within an equation and numerical values matching these terms. While the proposed approaches and guidelines sometimes conflict (e.g., limiting usage to four colors at most would make some of the proposals difficult to implement), the feedback is valuable as a first step toward formulating a more consistent use of color. 


\section{Video resolution}

How picky are the students when it comes to the resolution of the screen-captured videos? The answer to this question has several practical implications with respect to content production, storage, and delivery. Should students not be so concerned about resolution negatively affecting their learning, screencast videos may be heavily compressed to decrease file size, which in turn would require less storage space on the content provider side and likely result in fewer issues with streaming to students. And if the content delivery is such that the material is downloaded onto a student's computational device (computer or tablet), then the question of on-board storage space can be an important issue. If the students believe very high-resolution videos are paramount to their learning, then the consequences of very large video files must be dealt with. Ideally there exists a balance between acceptable video resolution for learning and file size for on-site storage and/or streaming. Where do the students fall on the scale of acceptable video resolution?

While taking the online survey, the students were shown four short video clips identical in content but with varying video resolution. Identical screenshots from each of the four clips are shown in Figure 8.

The students were then asked to identify the clip with the minimum acceptable resolution with respect to their interest and learning. As shown in Figure 9, almost the same number of students reported that the clips with the highest (19 students, or 33\%) and second-highest (21 students, or $36 \%$ ) resolution constitute the minimum video quality before negatively affecting their interest and learning. Not too far behind is the clip with the second-lowest resolution (Clip 3), with 14 students (24\%) accepting this clip's video resolution as the minimum standard. What the authors conclude is that the students' learning would not very negatively be impacted if the screencaptured videos are exported with a resolution lower (that of Clip 2) than the highest available from Camtasia, thereby saving production time (exporting) and local storage space, but keeping in mind that video quality should not suffer to the point that artifacts are easily noticeable and distracting. 


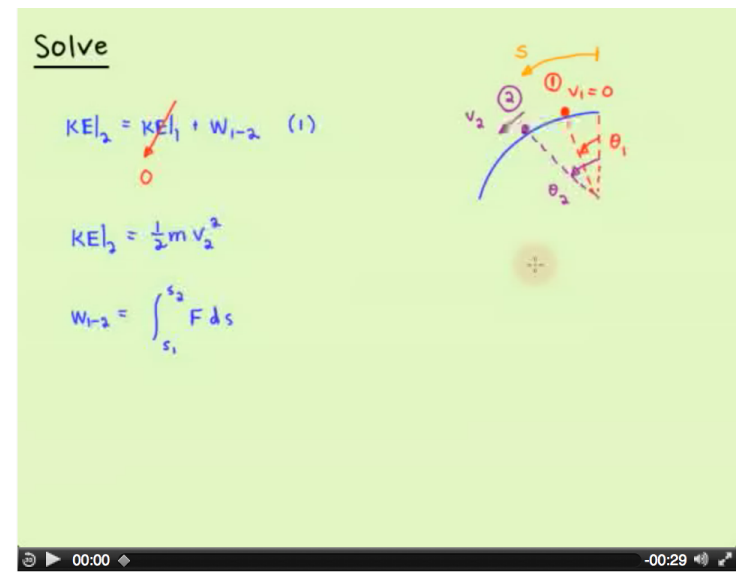

(a) Clip 1

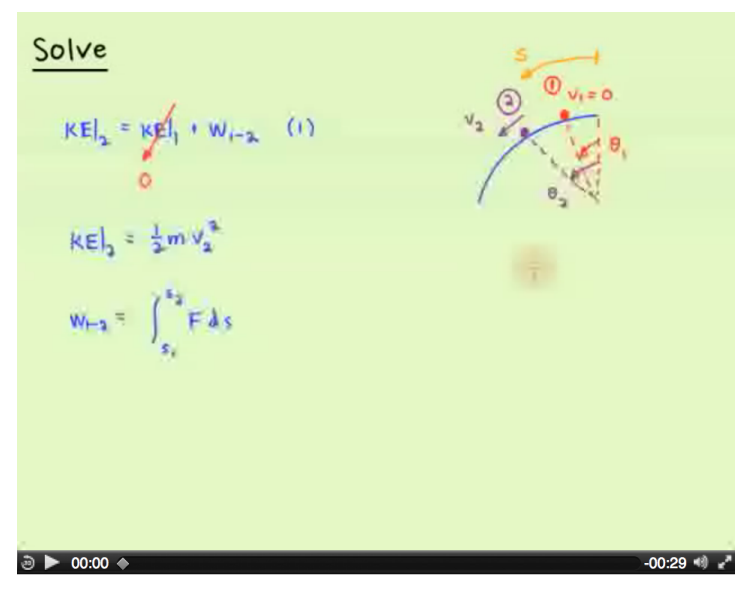

(c) Clip 3

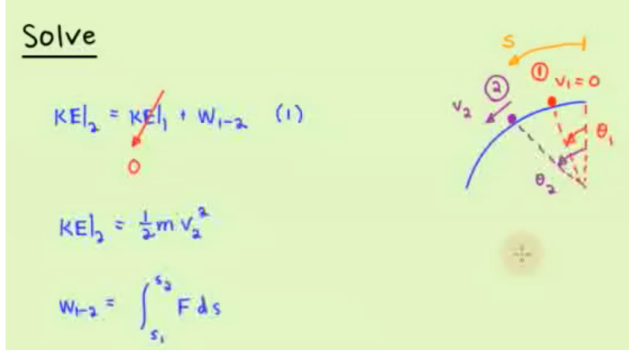

00:00

(b) Clip 2

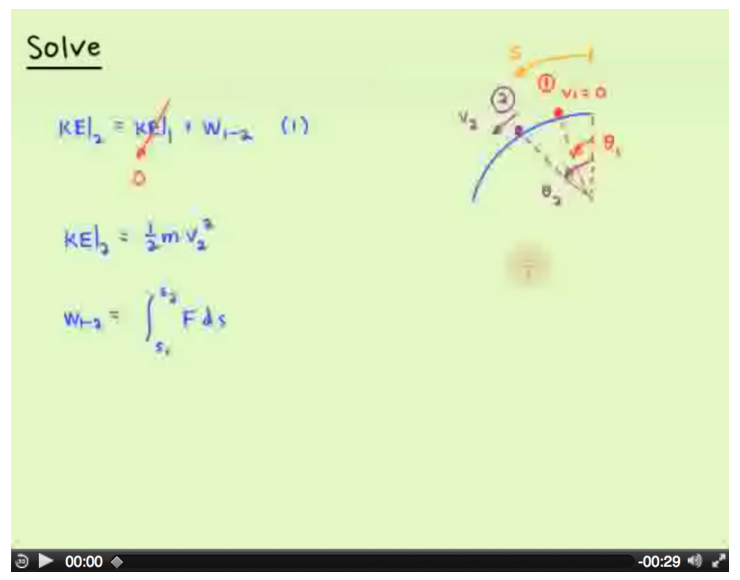

(d) Clip 4

Figure 8. Snapshots of the video clips used for asking students about the minimum acceptable video resolution with respect to their interest and learning. Clip 1 in $(a)$ has the highest resolution, and the resolution decreases in subsequent clips.

\section{Video length}

Lastly, with all the media attention given to massive open online courses (MOOCs) in recent years, there has been and continues to be much discussion about the "optimal" length of online videos with respect to student viewing retention and learning. Some of this discussion is informal and anecdotal ${ }^{8}$, but it serves to show that, based on how various MOOCs are structured, there seems to be little consensus on just how long online video content should be, whether the content is lecture-based or contains supplemental information like example problems. Some MOOC providers are partnering with researches to engage in formal studies to better understand video length and how it affects student viewing ${ }^{9,10}$. 
Clearly one cannot obtain "the" answer with such a limited study but, nevertheless, the authors felt they would be remiss to not address the issue, even if only to a limited degree. Thus, it was decided to pose a simple question to our students in order to see how they reacted to the issue.

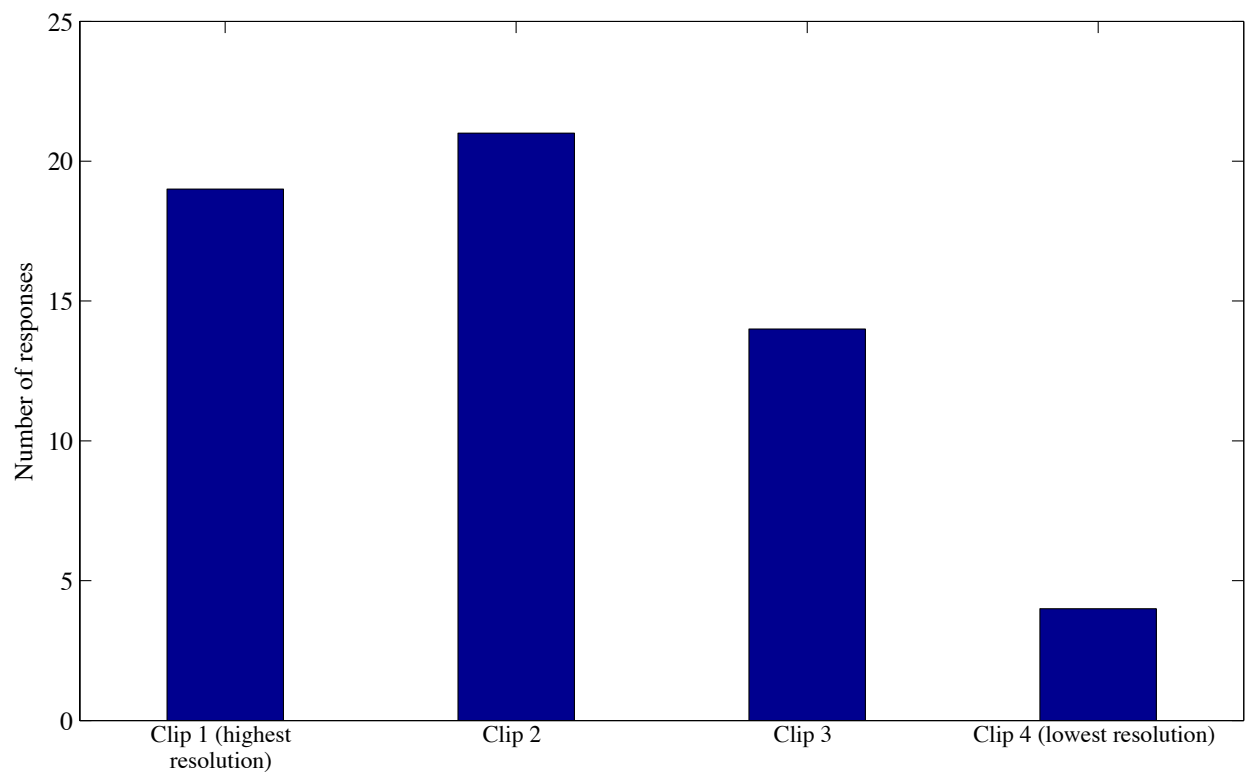

Figure 9. Responses to "Identify the clip with the minimum acceptable resolution with respect to your interest and learning." Students were asked to watch four short video clips, all identical except for the video resolution.

When asked about the importance of the length of each video lesson clip with respect to their learning, the students responded that the video length is important (46 students, or $79 \%$ ), according to the results in Figure 10. However, the students were split over the level of importance, with a majority (52\%) of these students claiming video length to be only slightly important, while the remaining $48 \%$ were split between rating the video length as moderately or very important.

With a majority of students claiming that video length is only slightly important to their learning and another 20\% (12 students) with either no opinion or believing video length to be unimportant, an initial conclusion from the results may be that video length is not as important as some may make it out to be. However, the authors realized after conducting the survey that the language in the question was imprecise, potentially leading the students to have posed a different question to themselves than had been intended. Specifically, the wording "video lesson clip" may have been interpreted as either each sub-clip for the entire video lesson (which was the intended interpretation because each example video was split into smaller clips [no more than 4 minutes] for, presumably, easier "digestion") or as the video lesson in its entirety. In other 
words, the word "clip" had not been precisely defined for them. Thus, if one were limited to the numerical data, one might hesitate to draw any firm conclusions.

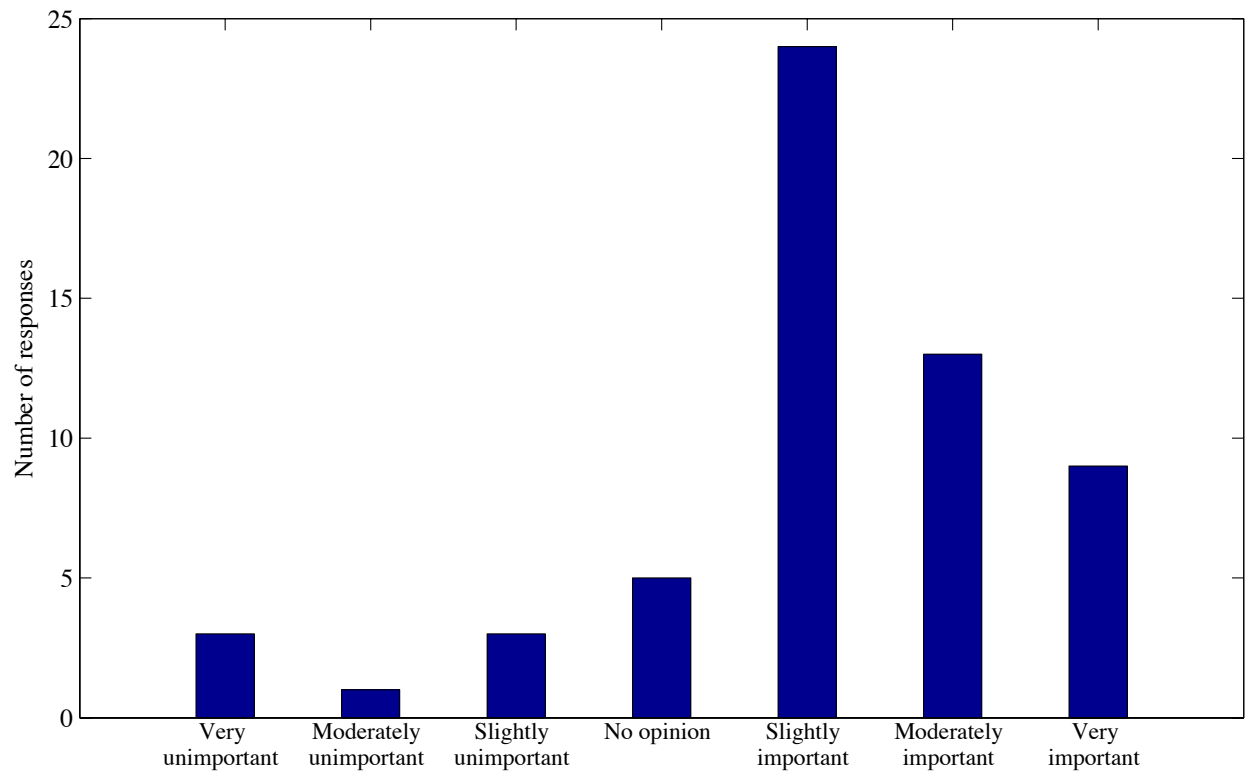

Figure 10. Responses to "How important is the length of the video lesson clip with respect to your learning?"

Fortunately, when students were free to comment at the end of the survey on aspects of the screen-captured example videos, many discussed the length of the videos and provided insightful feedback:

- "The video was relatively long but I can see how it can be useful for a student who is just getting introduced to the material."

- "The length of the video that I would be able to pay attention to depends on how interesting the subject is to me so that might vary."

- "Create a subtitled option with variable playing speeds. So, for example, I could play the video at $1.5 \mathrm{x}$, if I could read subs that fast."

- "Generally I think the videos could have a bit faster pace ..."

- "It would be nice to have an option to speed up or slow down the video (e.g. 1.2, 1.5, or $2 \mathrm{x}$ the normal speed) so that it would still be possible to watch the whole video but at a more comfortable pace for the viewer." 
- "It doesn't have to go super slow because the viewer can pause the video."

- "They should be a bit faster and more concise. There's no need to repeat anything on a video because the person can always rewind it and check what was said if they need to."

Based on these comments, the authors could conclude that the students' preference is for shorter, faster paced, and more concise videos. At the same time, the request for playback controls that allow the students to view the videos at, say, $1.5 \times$ (and we do intend to offer this ability in the final product) seems to make this preference a moot point. Indeed, with such playback controls available, would it even matter how long the screen-captured examples are if students (especially the more advanced ones) are always free to scrub through the content at a more comfortable pace? Do suggested rules of thumb for shorter video length, say, 6 to 9 minutes $^{9}$, still apply? Moreover, with these playback controls, is there still a perceived benefit from splitting a single example video into shorter clips when students may easily view the video in its entirety at a pace to their liking? Is it even good pedagogical practice to split a tutorial-type video?

One possibility that grows from the student commentary is to consider constructing the videos along lines analogous to computer hypertext. There could exist a bare-bones video with text and commentary that covers the material, but if a user clicked for "more information" within the video, it would bring up a more nuanced and in-depth exposition. This interactivity would then make the video more akin to a lecture, in which a student could ask questions which would prompt the instructor to pause his or her presentation and go into more depth regarding the particular point under consideration.

\section{Conclusion}

The authors have presented the results of a survey in which students rated and commented on the importance of various aspects of screen-captured example videos with respect to their learning and comprehension: separation of a solution process into smaller steps, explanatory narration, use of multiple colors for text elements, video resolution, and video length. Some of these aspects are pedagogical practices that carried over from the traditional print medium, while others are unique to the digitalization of learning that is sweeping higher education. All aspects, however, affect not only video content production and delivery on the instructor and content provider side, but viewership, interest, and learning on the student side. The voice of the student should play some role in determining how content is produced and delivered, as the student is the end user. 
Based on our results, the authors have drawn the following conclusions:

1. In the transition from text to video, students continue to respond favorably to the practice of separating the solution process for an example problem into steps that are clearly delineated, and thus this practice should be encouraged in creating screen-captured example problems or tutorials.

2. While including in-depth explanatory narration is widely and highly valued by the students with respect to their learning, comments reveal that this may be at odds with a desire for shorter videos. Perhaps a compromise may be to remove from a video what students might perceive as "extraneous" explanatory narration and make it supplementary exposition (either in text or audio format) available on demand during the course of the video. A comparison of the effectiveness of the videos as they stand and with this revised format would make for an interesting study, especially since students have a tendency to skip anything that is not required.

3. From a stylistic standpoint, students strongly support the use of different colors for different text and graphical elements during the solution process. Some students opined that, while the use of color is an important stylistic aspect, it does little to enhance their learning, but a clear majority think otherwise, explaining that different colors help to highlight important and distinct parts of the overall solution process. The authors encourage targeted use of color in screen-captured videos.

4. Overall, students do not appear to be especially concerned with respect to video resolution. It is not necessary to export videos at the highest data rate available, but some care is needed to balance savings in video exporting time and local storage space (on both the content provider side for streaming and on the consumer side if content is to be disseminated via download) with maintaining a certain level of video quality that does not negatively affect student learning. There is another important point to keep in mind. This not-so-picky attitude might be a product of the younger generations' interaction with YouTube and other free video streaming services, where one could argue that availability of video may be more important to viewers than quality. Since these screen-captured example videos will be part of a dynamics learning platform that students will pay for, even though students say they could adequately learn from lower resolution videos, they may espouse higher video quality standards because they are paying for the content.

While no firm conclusions may be drawn from the students' rating of video length because of some ambiguity in the phrasing of the posed question, solicited comments seem to imply that the benefit of shorter videos may be a moot point in the face of playback controls that allow students to watch a video, no matter its length, at whatever pace is to their liking. The authors look 
forward to seeing future studies, along the lines of the one conducted by Kim et al. ${ }^{10}$, that would examine student viewing patterns when having the option to watch a video at $1.5 \times, 2 \times$, etc., and eventually link those patterns to learning and retention.

\section{References}

[1] N. Halyo and Q. Le, "Use of video technology to improve student learning," 2013 ASEE Annual Conference \& Exposition, Atlanta, GA, USA, June 2013.

[2] T. Kunberger and K. R. Csavina, "Work it baby, work it! Reworking the worksheet in Camtasia," 2011 ASEE Annual Conference \& Exposition, Vancouver, BC, Canada, June 2011.

[3] S. Moseley and S. M. Sipes, "Initial investigation in the effect of homework solution media on fundamental statics comprehension,” 2013 ASEE Annual Conference \& Exposition, Atlanta, GA, USA, June 2013.

[4] G. L. Gray, F. Costanzo, and M. E. Plesha, "Problem solving in statics and dynamics: A proposal for a structured approach,” 2005 ASEE Annual Conference \& Exposition, Portland, OR, USA, June 2005.

[5] F. Costanzo and G. L. Gray, "A structured approach to problem solving in statics and dynamics: Assessment and evolution,” 2008 ASEE Annual Conference \& Exposition, Pittsburgh, PA, USA, June 2008.

[6] S. Moseley, "Getting started with screencasting: A tool to supplement classes, answer student questions, and provide guided analysis practice," 2013 ASEE Annual Conference \& Exposition, Atlanta, GA, USA, June 2013.

[7] F. Oncul, "Video lecture capture in engineering classrooms with free open-source software tools," 2011 ASEE Annual Conference \& Exposition, Vancouver, BC, Canada, June 2011.

[8] Degree of Freedom [Jonathan Haber], "MOOC Components - The Lecture," Degree of Freedom. Degree of Freedom. March 18, 2013. Web. January 1, 2014.

[9] Phillip Guo, “Optimal Video Length for Student Engagement,” edX Blog, edX. October 29, 2013. Web. January 1, 2014.

[10] J. Kim, P. J. Guo, D. T. Seaton, P. Mitros, K. Z. Gajos, and R. C. Miller, "Understanding in-video dropouts and interaction peaks in online lecture videos," submitted for the ACM Conference on Learning at Scale, Atlanta, GA, USA, March 2014. 